\section{Rumning missing scan: Perception of oldest member in serial presentations}

JAMES $V$. HINRICHS, University of Iowa, Lowa Citv, lowa 52240, and HERMAN BUSCHKE, Albert tinstein College of Medicine, Eastchester Rd. and Morris Park Ave., Bronx, N.Y. 10461

The ability to select the current oldest item in a homogeneous series of items was examined in 250-item sequences with an "age" range of 1-15 items. Approximately $50 \%$ of the responses were correct, and the probability of a correct response increased linearly with the age of the correct response. The memory requirements of the task were discussed and compared with other covert retention tasks.

Several recent studies have been concerned with evaluating human memory performance in situations where the retention measure is derived indirectly. If standard memory performance measures, such as recall and recognition, can be considered overt measures of retention. then these indirect assessments might be termed "covert" retention. In an overt memory task, retention is tested by requiring the $S$ to respond with the item or items that have been presented previously. In a covert memory task, the S's task is to avoid responding with an item that has been presented. For example, in the missing scan (Buschke, 1963), the $S$ is presented with $\mathrm{N}-1$ members of a well-known class of $\mathrm{N}$ items and asked to respond with the unpresented or missing item. With the guessing technique (Hinrichs, 1966), the $S$ is required to respond before each item is presented, always attempting to respond with an item not yet presented. In both tasks, the evaluation of retention performance is indirect: The better the $S$ successfully avoids responding with presented items, the better is his memory.

One way to characterize the S's performance in covert retention tasks is to consider the S's problem as one of attempting to respond with the "oldest" item, that is, the least recent item presented. Then the S's problem reduces to one of attempting to judge the current oldest member of his response set. Conversely, the $S$ avoids responding with the items judged to have been presented recently.

Judgment of recency (JOR) has been studied as a task in discrimination (Yntema \& Trask, 1963), and as an absolute judgment task in short lists (Peterson,
1967) and in steady-state situations (Hinrichs \& Buschke, 1968). In the steady-state paradigm, a small set of items is repeatedly presented in a random order. As each successive presentation is made, the $S$ is required to make a numerical estimate of the number of intervening items since its last presentation. When these judgments are compared with the actual distances, or lags, it is found that Ss overestimate the recency of short lags and underestimate the recency of longer lags in the range of lags from 2 to 15 .

The JOR task is closelv related to the discrimination of oldness required in the covert memory tasks. In the present study, the same steady-state presentation paradigm used in the Hinrichs \& Buschke (1968) experiment was employed to ask a different question. However, instead of numerical estimates of recency for each item, the $S$ was required to respond with the item he perceived as being the current "oldest" item in the set available to him. This task more nearly resembles the retention requirements of covert memory tasks than does the JOR task. It is especially similar to the missing scan (Buschke, 1963) in that the $S$ is required to respond with the least recent member of the set, i.e., the "least remembered" item. Unlike the missing scan paradigm, however, the current procedure employs very long sequences and continuous judgments on the part of the $S$, hence the paradigm can be considered a "running missing scan."

\section{METHOD}

The materials and procedures used were identical in most respects to those used in Hinrichs \& Buschke (1968), which should be consulted for further details. A summary of the method and discrepancies from the earlier study are reported here. Materials and Apparatus

The stimuli were eight letters of the alphabet: $Q, R, S, T, W, X, Y$, and $Z$. The letters were approximately evenly distributed within a sequence of 250 presentations so that the interpresentation intervals (lags) were approximately rectangularly distributed. Hence, each one of the 15 possible lags from 1 to 15 occurred approximately equally often. Eight sequences of 250 presentations were constructed and presented to each $S$, with the order of presentation counterbalanced across Ss.

The stimuli were displayed on an Industrial Electronics Engineering Bina-View cell programmed by a punched paper tape reader. The programming and presentation equipment were isolated from the $S$ in a soundproof chamber. The $S$ viewed the display cell through the window of an adjoining soundproof chamber; his verbal responses were recorded by $E$.

\section{Subjects}

The eight Ss were paid for their participation. Each $S$ was tested individually and served in three 1-h sessions, with each session separated by at least 1 day.

After each successive letter appeared, the $S$ was instructed to respond with the letter that he believed to be the current "oldest" letter, i.e., the letter with the longest interval since its last presentation. Several examples were shown to the $S$, and a short practice session was administered to ensure S's understanding of the experimental requirements. The first session then concluded with the presentation of two 250 -letter sequences at a 3-sec presentation rate. In each of the other two sessions, three sequences were presented. The first 10 responses were removed from the response protocols, with the eight Ss producing 240 observations for each of the eight sequences for a total of 15,360 observations.

\section{RESULTS}

Of the 15,360 responses produced by the eight Ss, there were 7,421 instances in which the current oldest letter was the S's response and 7,939 instances in which it was not. Hence, the proportion of correct identification of the current oldest member of the stimulus set was .483 .

To evaluate further the quality of the Ss' performance, it is first necessary to consider the relevant characteristics of the sequences presented to them. The interval since the last presentation of any particular item, $\mathbf{i}$, was considered that item's age, $\mathbf{A}_{\mathbf{i}}$. At any given point in the sequence, after each of the items had been presented at least once, one of the items was the current oldest item. That is, at any point in the sequence there was one item, 0 , in the sequence such that for any other item, $i$, in the set, $A_{o}>A_{i}, \quad o \neq i$. The range of possible values for $A_{0}$ was 8 to $15 ; 15$ was the maximum age possible as the distribution of items was constructed, and, because there were eight members of the set, any item that was older than all the others must have had an age of at least 8 . For the present set of items, the overall mean age, i.e., the mean $A_{i}$ of all items available to the $S$, was 5.74 . The mean age of the oldest item, $\bar{A}_{0}$, was 11.92 .

The mean age of all the responses produced by the Ss was 10.06. The mean age of the correct responses was 12.20 $(S D=1.76)$; of the incorrect responses, 
Fig. 1. Probability of choosing the oldest item as a function of the age of the oldest item. (The linear approximation is fit by eye.)

i.e., instances in which there existed at least one member of the set older than the response, $\bar{A}_{i}=8.08(\mathrm{SD}=2.33)$. When the $S$ made his response, the letter he chose was older than some number of the scven other possibilities. A correct response represents an item that is older than all seven alternatives and occurred $48.3 \%$ of the time. The mean number of items rejected was 6.00 .

Many of the correct responses occurred during runs of the same response item. The 15,360 responses can be represented as 3,895 runs of one or more consecutive responses of the same item. The mean run length was 3.94 items, with $51.1 \%$ of the runs composed of three or fewer items.

As the age of the correct response increases, the probability of the correct response being chosen by the $S$ also increases (Fig. 1). When the age of the oldest item is 8 , the probability of selecting it was only .344 , but this value increased to .618 when the age was 15 .

\section{DISCUSSION}

The ability to perceive the current oldest item with good precision in the current study is consistent with the recent investigations of judgment of recency (Hinrichs \& Buschke, 1968; Lockhart, 1969; Peterson, 1967; Yntema \& Trask, 1963), and suggests the operation of the same processes in both cases. When the $S$ is confronted with the task of selecting the current oldest item, he may proceed by first attempting to eliminate the most recently presented items. The evidence in the present study suggests that the $S$ is usually able to eliminate about six of the eight items in the stimulus set. The S's candidate for the current oldest item is then selected from the items not eliminated and given as the response until it occurs. This strategy would appear to

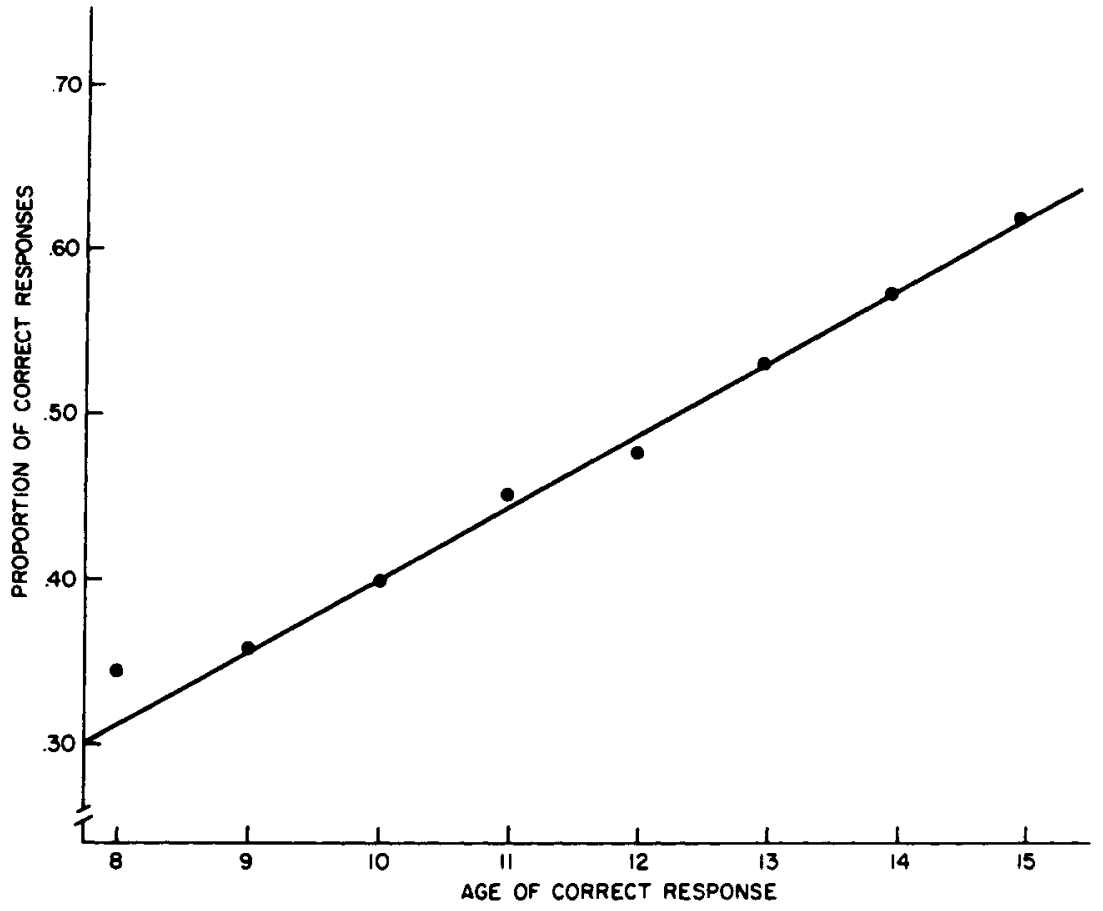

maximize the likelihood of giving the oldest item within the constraints of the $S$ 's memory ability. In any case, when the $S$ persists with one item until it does occur, the response becomes older with each succeeding presentation, increasing both the mean age of the response and the likelihood of its being the current oldest item.

The linearity in the relationship between the age of the oldest item and the probability of correctly selecting the oldest item also is probably due to the fact that responses occur in runs. A response is produced until it occurs, increasing the probability that it will be the oldest item. Since the distribution of lags is approximately rectangular, the mean increase in the probability of the selected response being correct would be approximately constant.

\section{REFERENCES}

BUSCHKE, H. Retention in immediate memory estimated without retrieval. Science. 1963, $140,56-57$.

HINRICHS. J. V. Short-term memory with a guessing technique. Journal of Experimental Psychology, 1966, 71, 89-95.

HINRICHS, J. V., \& BUSCHKE. H. Judgment of recency under steady-state conditions. Joumal of Experimental Psychology. 1968, 78. 574-579.

LOCKHART, R. S. Recency discrimination predicted from absolute lag judgments. Perception \& Psychophysics, 1969, 6. 42-44.

PETERSON, L. R. Search and judgment in memory. In B. Kleinmuntz (Ed.), Concepts and the structure of memory. New York: Wiley, 1967. Pp. 153-180.

YNTEMA, D. B., \& TRASK, F. P. Recall as a search process. Journal of Verbal Learning \& Verbal Behavior, 1963, 2, 65-74. NOTES

1. This investigation was supported in part by PHS Grants No. MH-16362 to the first author and MH-08556 to the second author from the National Institute of Mental Health. 